\title{
Mapping major quantitative trait loci for postnatal growth in an intersubspecific backcross between C57BL/6J and Philippine wild mice by using principal component analysis
}

\author{
Akira Ishikawa* and Takao Namikawa \\ Laboratory of Animal Genetics, Division of Applied Genetics and Physiology, \\ Graduate School of Bioagricultural Sciences, Nagoya University, Chikusa-ku, \\ Nagoya, Aichi 464-8601, Japan
}

(Received 19 September 2003, accepted 3 February 2004)

\begin{abstract}
A number of quantitative trait loci (QTLs) for postnatal growth have previously been reported in mice. As effects of the QTLs are usually small and similar to one another in magnitude, it is generally difficult to know which loci are major contributors to postnatal growth. We applied principal component analysis to a genome-wide search for QTLs affecting postnatal growth in body weight weekly recorded from 3 to 10 weeks of age in an intersubspecific backcross population of C57BL/6J inbred mice (Mus musculus domesticus) and wild mice (M. m. castaneus) captured in the Philippines, in order to discover new QTLs from a gene pool of the wild mice and uncover major loci underlying variation in postnatal growth. Principal component analysis classified phenotypic variation in body weights at different ages into two independent principal components: the first principal component (PC1) extracted information on the entire growth process and the second principal component (PC2) contrasted middle (3-6 weeks of age) with late (6-10 weeks) growth phases. Simple interval mapping and composite interval mapping revealed 10 significant QTLs with main effects on PC1 or PC2 on eight chromosomes. Of these, the six main-effect QTLs interacted epistatically with one another or three new additional QTLs on different chromosomal regions without main effects. Several of the identified QTLs with main effects and/or epistatic interaction effects appeared to be sex specific. These results suggest that the identified 13 QTLs, most of which affected the entire growth process, are very important contributors to complex genetic networks of postnatal growth.
\end{abstract}

Key words: body weight, epistasis, quantitative trait loci, sex specificity, wild mouse

\section{INTRODUCTION}

Quantitative variation is ubiquitously observed in a great number of traits such as agriculturally important traits and common human diseases. Since late 1980s, quantitative trait locus (QTL) mapping has become a standard tool for unraveling the genetic basis of the variation. In fact, many QTL mapping studies have identified the genomic regions contributing to a variety of quantitative traits in animals and plants (Andersson 2001, Flint and Mott 2001, Mackay 2001). Very recently, several QTLs have positionally been cloned (e.g.,

Edited by Toshihiko Shiroishi

* Corresponding author. E-mail: ishikawa@agr.nagoya-u.ac.jp
Frary et al. 2000, Grisart et al. 2001), heralding a new era in which the molecular characterization of a QTL will become standard practice to do. However, the actual identity of polymorphism(s) responsible to the QTL effect has still remained unknown, with a few exceptions (Liang et al. 2003).

Body weight has long been used as a model quantitative trait in the mouse to understand its genetic architecture because of the ease of measurements throughout life with great accuracy and reliability. Many QTL mapping studies have so far located a number of QTLs affecting body weight and growth-related traits on almost all chromosomes in mice and have revealed that the magnitude of each QTL effect is usually small with the total phenotypic variance of $\sim 15 \%$ and not greatly different from one 
another (e.g., Cheverud et al. 1996, Keightley et al. 1996, Brockmann et al. 1998, Morris et al. 1999, Vaughn et al. 1999, Brockmann et al. 2000, Cheverud et al. 2001, Reed et al. 2003). A QTL for 6-week body weight accounting for up to $35 \%$ of the phenotypic variance (Brockmann et al. 1998) and a X-linked QTL with an approximately $20 \%$ effect on growth rate (Rance et al. 1997a, 1997b) are reported as exceptional cases. Despite the existence of a large number of the QTLs with small effects, a rather small number of loci are likely to contribute greatly to complex physiological pathways that regulate growth and obesity. Until now, fewer studies have attempted to specify such key loci. As information about genes at those loci can readily be extrapolated from mice to farm animals and humans, it will help to perform the efficient improvement of meat quality or carcass traits in farm animal breeding and also to provide effective medical treatments for growth disorders or obesity in humans.

It is well known that, as most laboratory mouse strains commonly used in the world are derived from a relatively small pool of ancestors, their genetic polymorphisms are probably very limited (Nishioka 1995, Guenet and Bonhomme 2003). We have previously mapped several QTLs with main effects and/or epistatic interaction effects on adult body weight at 10 weeks after birth in an intersubspecific backcross population derived from a cross between the standard inbred strain C57BL/6J (Mus musculus domesticus) and wild mice (M. m. castaneus) captured in the Philippines (Ishikawa et al. 2000). Some of the mapped QTLs are novel or have unique effects, suggesting that the utilization of wild animals are important as new sources of valuable QTLs in animals.

In the present study, we extended the above QTL mapping of a single trait obtained at one age to a QTL mapping experiment for multiple body weight traits obtained by consecutive observations from 3 to 10 weeks of ages. In order to find additional new growth QTLs from a gene pool of the wild mice and to uncover key loci underling variation in postnatal growth, we applied principal component analysis to the genome-wide QTL search because of two reasons. First, we can easily perform multipletrait QTL analysis with both a general statistical package containing principal component analysis and widely-distributed, user-friendly QTL mapping software without building difficult models. Second, principal component analysis is known to be a good statistical method for extracting a few, informative and independent principal components from information on many measurements that are often highly correlated with one another, without losing much information. These principal components have usually biological meanings and can be subjected to QTL mapping as composite traits. Statistical properties of this approach have been investigated and validated (Weller at al. 1996, Mangin et al. 1998). In fact, it is proven that the genetics of principal components can be used for dissection of genetic networks that regulate complex biological systems, such as colitis susceptibility in mice (Farmer et al. 2001; Mähler et al. 2002) and skeletal variation in pigs (Andersson-Eklund et al. 2000) and dogs (Chase et al. 2002).

\section{MATERIALS AND METHODS}

Animals. The construction method of the mapping population used in this study and the details of animal husbandry are described by Ishikawa et al. (2000). Briefly, a pair of adult wild mice at unknown ages were captured live in Luzon Island, the Philippines in June 1994 and introduced into our laboratory. The four wild males obtained from a cross of this pair were mated with eight females of the C57BL/6J inbred strain purchased from Clea Japan, Inc. (Tokyo). Thirty-four females at $\mathrm{F}_{1}$ generation were repeatedly backcrossed to their own wild male parents. Finally, a total of 387 backcross mice (186 males and 201 females) from 70 first to third parity litters were produced. Litter size was not standardized at birth to maximize the number of backcross mice reared. Pups were weaned at 3 weeks after birth. Commercial food and tap water were provided ad libitum.

Genotyping. Primer pairs of mouse microsatellite markers were purchased from Research Genetics Inc. (Huntsville, Ala). As described in Ishikawa et al. (2000), a total of 90 fully informative markers were selected (Table 1). Genomic DNA was extracted from kidneys by standard methods. PCR amplification of microsatellite markers was performed using the procedure of Routman and Cheverud (1994). PCR products were electrophoresed on $3.5 \%$ agarose gels and visualized by ethidium bromide staining. Doubtful marker genotypes were retyped.

Linkage maps. Three linkage maps were constructed from male, female and sex-pooled data with the computer software Map Manager QTXb17 (Manly et al. 2001). The recombination frequencies (\%) were converted into genetic distances in $\mathrm{cM}$ using the Kosambi map function. The average marker spacing was $20.2 \mathrm{cM}$ in male-specific, $19.5 \mathrm{cM}$ in female-specific and $19.8 \mathrm{cM}$ in the sex-averaged linkage maps.

Body weight measurements. The body weights of the backcross mice were recorded weekly from 3 to 10 weeks of age to the nearest $0.1 \mathrm{~g}$ with a digital balance. Effects of four environmental factors, sex, parity, litter and litter size, on eight body weights were tested by the FIT Y BY X PLATFORM of JMP software Version 5.01J (SAS Institute, Inc., Cary, NC). Litter was treated as a random effect and the remainders were treated as fixed effects. All possible two- and three-way interactions of the fixed effects were also included in the model. Although the 
Table 1. The 90 microsatellite markers genotyped in this study.

\begin{tabular}{|c|c|c|c|c|c|c|c|}
\hline Marker & Pos. & Marker & Pos. & Marker & Pos. & Marker & Pos. \\
\hline D1Mit3 & 11.0 & D6Mit138 & 0.7 & D11Mit1 & 0.3 & D16Mit $32^{\mathrm{ab}}$ & 1.7 \\
\hline D1Mit71 & 19.5 & $D 6 M i t 74^{\mathrm{a}}$ & 20.5 & D11Mit24 & 27.9 & D16Mit35 & 14.0 \\
\hline$D 1 M i t 9^{\mathrm{ab}}$ & 52.0 & $D 6 M i t 29^{\mathrm{ab}}$ & 36.5 & $D 11 M i t 36^{\mathrm{ab}}$ & 47.6 & D16Mit49 $9^{\text {ab }}$ & 53.0 \\
\hline \multirow[t]{2}{*}{$D 1 M i t 15^{\mathrm{a}}$} & 87.9 & D6Mit36 & 46.0 & $D 11 M i t 67^{\mathrm{a}}$ & 57.0 & D16Mit51 $1^{\mathrm{a}}$ & 66.8 \\
\hline & & $D 6 M i t 12^{\mathrm{ab}}$ & 59.6 & D11Mit104 & 79.0 & & \\
\hline$D 2 M i t 1^{\mathrm{ab}}$ & 1.0 & $D 6 M i t 201^{\mathrm{a}}$ & 74.1 & & & $D 17 M i t 48^{\mathrm{ab}}$ & 5.0 \\
\hline D2Mit293 & 11.0 & & & ${ }_{D 12 M i t 103^{\mathrm{a}}}$ & 1.0 & D17Mit118 & 37.7 \\
\hline$D 2 M i t 7^{\mathrm{a}}$ & 28.0 & $D 7 M i t 74^{\mathrm{a}}$ & 2.0 & D12Mit54 ${ }^{\mathrm{a}}$ & 24.0 & D17Mit123 & 56.7 \\
\hline D2Mit324 & 32.8 & D7Mit83 & 26.5 & D12Mit4 & 34.0 & & \\
\hline D2Mit $15^{\mathrm{a}}$ & 50.0 & D7Mit5 & 46.4 & $D 12 N d s 2$ & 59.0 & D18Mit $19^{\mathrm{a}}$ & 2.0 \\
\hline$D 2 M i t 48^{\mathrm{a}}$ & 87.0 & D7Mit68 & 60.0 & & & $D 18 M i t 90^{\mathrm{a}}$ & 28.0 \\
\hline \multirow[t]{2}{*}{$D 2 M i t 266^{\mathrm{ab}}$} & 109.0 & $D 7 M i t 259^{\mathrm{a}}$ & 72.0 & D13Mit84 ${ }^{\mathrm{a}}$ & 11.0 & D18Mit196 & 58.0 \\
\hline & & & & D13Mit $26^{\mathrm{a}}$ & 38.0 & & \\
\hline$D 3 M i t 93^{\mathrm{ab}}$ & 13.8 & $D 8 M i t 141^{\mathrm{ab}}$ & 6.0 & D13Mit195 & 51.0 & D19Mit29 ${ }^{\mathrm{a}}$ & 4.0 \\
\hline D3Mit184 & 28.0 & $D 8 M i t 30^{\mathrm{a}}$ & 33.0 & D13Mit150 & 71.0 & D19Mit97 ${ }^{\mathrm{a}}$ & 22.0 \\
\hline$D 3 M i t 12^{\mathrm{a}}$ & 49.2 & D8Mit154 & 59.0 & $D 13 M i t 77^{\mathrm{a}}$ & 73.0 & $D 19 M i t 13^{\mathrm{a}}$ & 33.0 \\
\hline D3Mit14 ${ }^{\mathrm{a}}$ & 64.1 & & & & & $D_{19 M i t 4^{\mathrm{a}}}$ & 48.0 \\
\hline \multirow[t]{2}{*}{ D3Mit19 } & 87.6 & $D 9 M i t 2^{\mathrm{a}}$ & 17.0 & $D 14 M i t 179^{\mathrm{a}}$ & 2.5 & $D 19 M i t 33^{\mathrm{ab}}$ & 53.0 \\
\hline & & D9Mit340 & 41.0 & D14Mit159 & 30.0 & & \\
\hline$D 4 M i t 93^{\mathrm{a}}$ & 10.6 & D9Mit35 & 52.0 & $D 14 M i t 35^{\mathrm{a}}$ & 44.0 & DXMit124 & 2.8 \\
\hline D4Mit15 & 42.6 & D9Mit18 & 71.0 & $D 14 M i t 267^{\mathrm{a}}$ & 60.0 & DXMit57 & 5.9 \\
\hline \multirow[t]{2}{*}{$D 4 M i t 32^{\mathrm{a}}$} & 69.8 & & & & & DXMit75 & 18.9 \\
\hline & & D10Mit $75^{\mathrm{a}}$ & 2.0 & D15Mit $12^{\mathrm{a}}$ & 4.7 & DXMit19 & 43.2 \\
\hline$D 5 M i t 345^{\mathrm{ab}}$ & 1.0 & $D_{10 M i t 16}{ }^{\mathrm{a}}$ & 16.0 & $D 15 M i t 6^{\mathrm{a}}$ & 13.7 & DXMit122 & 72.5 \\
\hline D5Mit4 $^{\mathrm{a}}$ & 20.0 & D10Mit20 & 35.0 & D15Mit29 & 42.8 & & \\
\hline$D 5 M i t 5^{\mathrm{ab}}$ & 36.0 & D10Mit244 & 55.5 & D15Mit $34^{\mathrm{a}}$ & 52.2 & & \\
\hline$D 5 M i t 9^{\mathrm{a}}$ & 54.0 & D10Mit145 & 70.0 & & & & \\
\hline$D 5 M i t 326^{\mathrm{ab}}$ & 78.0 & & & & & & \\
\hline$D 5 M i t 51^{\mathrm{ab}}$ & 81.0 & & & & & & \\
\hline
\end{tabular}

Pos., Map positions in cM were taken from the mouse consensus map (the Mouse Genome Database: MGD).

${ }^{a}$ Markers with multiple alleles within wild mice.

${ }^{\mathrm{b}}$ Wild-derived multiple alleles not separable from one another with absolute confidence under our electrophoresis condition.

fixed and random effects significant at the nominal 5\% levels were somewhat different depending on weights, all raw weights were corrected by the same set of the fixed effects of sex and litter size and the random effect of litter in order to interpret the final results with consistency.

Principal component analysis. After Pearson product-moment correlations for each pair of the eight corrected body weights were computed, principal component analysis was performed using the correlation matrix of the MULTIVARIATE PLATFORM of JMP. The number of principal component axes to retain was determined by the scree test (Dillon and Goldstein 1984). Loadings for body weights were calculated by multiplying a square root of eigenvalues by standardized eigenvectors. The principal components summarizing the information from the eight weights were used as univariate traits for QTL analyses below.

Interval mapping. Alleles at many marker loci used here were not fixed within the wild mice (Table 1). Before interval mapping, to determine whether multiple QTL alleles were segregating in the wild mice, simple associations between genotypes and principal component scores were assessed for each polymorphic marker locus by one-way analysis of variance (ANOVA) using the FIT Y BY X PLATFORM of JMP.

Two interval mapping methods were used in this study. One is simple interval mapping (SIM) with a maximum-likelihood method (Lander and Botstein 1989) 
based on a single QTL model. The other is composite interval mapping (CIM) based on a multiple QTL model. Both SIM and CIM were carried out with the computer software QTL Cartographer Version 1.14d (Basten et al. 2000). By combining SIM with multiple regression, CIM can localize QTLs more precisely than does SIM because of controlling the genetic background containing the other QTLs, and also can control for spurious ghost loci (Zeng 1993, 1994, Doerge et al. 1997, Doerge 2002). Furthermore, CIM has recently been validated its usefulness for detection of multiple linked, sex-specific QTLs (Butterfield et al. 2003). This mapping uses two parameters: (1) the number of background markers to control for the genetic background; and (2) the window size for blocking out a region of the genome on either side of the markers flanking the test interval. The markers to control for the genetic background were chosen by forward/backward selection with an accepting/rejecting significance threshold of $1 \%$, as recommended by Zeng et al. (1999). The window size of $10 \mathrm{cM}$ was used according to our previous QTL study (Ishikawa et al. 2000).

The above SIM and CIM analyses were carried out with 2-cM steps within each interval. The estimates of map positions, likelihood ratio statistics, additive effects (differences between homozygotes and heterozygotes) and the proportion of the phenotypic variation explained by individual QTLs were computed by QTL Cartographer. LOD scores were obtained by dividing likelihood ratio statistics by 4.605 . For the $\mathrm{X}$ chromosome of males, the expected additive effect was a half the computed value because of its hemizygous state. The $95 \%$ confidence intervals for QTL positions were calculated from the formula of Darvasi and Soller (1997). The total contribution of all detected QTLs to the phenotypic variance was estimated by summing up the square of each additive effect and dividing it by 4 (Mather and Jinks 1977).

Empirical significance thresholds for both SIM and CIM were determined by permutation tests (Churchill and Doerge 1994) by using QTL Cartographer. One thousand permutations of our mapping data were carried out with 2-cM steps within intervals, and the computed threshold levels were evaluated using LOD scores.

Epistatic interaction. To identify QTLs with epistatic interaction effects, a genome-wide scan for all pairs of marker loci was performed with Map Manager QTX on the assumption that a QTL is right at a marker locus. This search is based on a linear regression model with a marker by marker interaction term and only provides likelihood ratio statistics for the overall and interaction effects in the model. The likelihood ratio statistic obtained was converted into a $P$ value by using a $\chi^{2}$ distribution with one or three degrees of freedom. The interaction effect and the percentage of the phenotypic variance explained by the interaction (the difference in variance between two models with and without the interaction term) were calculated by two-way ANOVA of JMP. The total contribution of all detected interactions to the phenotypic variance was estimated by multiple regression analysis of JMP.

To declare significant epistatic QTLs, two tests were carried out as described by Sen and Churchill (2001) and Chmielewicz and Manly (2002) with a slight modification. First, significance of the overall effect was established by 1000 permutations of Map Manager QTX. Second, if the overall effect exceeded the genomewide 5\% significance thresholds, then the interaction effect was tested using approximate genome-wide thresholds obtained as described by Knott et al. (1998). That is, the genome-wide thresholds computed by permutation test for the above SIM were directly used because both an interaction term and SIM had the same one degree of freedom. For confirmation, the interaction effect was also tested using the experiment-wise 5\% level $(P<0.05 /$ $18=0.0028)$ that was obtained by applying the Bonferroni correction for multiple tests. This two-stage test is needed because a strong main-effect QTL may lead to an increase of a likelihood ratio statistic for the overall effect even if the interaction effect is not significant (Chmielewicz and Manly 2002).

Sex specificity. To search for QTLs with sex-specific effects, the above SIM, CIM and epistatic interaction analyses were carried out for three sex-groups, male, female and sex-pooled, separately. For both SIM and CIM, it is impossible to investigate the interaction effect between QTL and sex using QTL Cartographer, because its interaction term can not be included in the model of the software (Basten et al. 2000). Thus, we were not able to perform a formal statistical test for sex specificity of QTL expression according to the method of Knott et al. (1998) and Quintanilla et al. (2002). To overcome this problem, a QTL was considered sex-specific with two circumstantial evidences. First was to show the evidence that, when a QTL exceeded genome-wide significance thresholds in one sex, the LOD score was 1.7 or more fold higher than that in the other sex. This LOD score difference corresponded to $P<0.05 / 10=0.005$ that was converted by using a $\chi^{2}$ distribution with one degree of freedom and obtained by applying for the Bonferroni correction of 10 fold. This approach was previously validated by Cheverud et al. (2001) and used by Bergeson et al. (2003) and Reed et al. (2003). A greater LOD score change might be need for CIM, but the actual value could not be determined as the number of background markers included in the model differed between sexes. The second evidence was to obtain the obvious difference in LOD score profiles along a chromosome between sexes.

By contrast, sex specificity for epistatic QTLs was tested by three-way ANOVA including an interaction 
term of an interacting marker pair and sex in the model because of the assumption described earlier. The Bonferroni-corrected experiment-wise 5\% level $(P<0.05 / 5=$ 0.01 ) was used as a significance threshold.

\section{RESULTS}

Phenotypic analysis. Phenotypic means and standard deviations, after correction for fixed and random effects, for eight body weights weekly measured from 3 to 10 weeks of age in the intersubspecific backcross population of C57BL/6J and Philippine wild mice are presented in Table 2. All pairwise correlations among the eight body weights were highly significant at $P<0.0001$ in the sexpooled group. These correlations declined when the difference in age between weights increased (Table 2). The same tendency was observed in each sex (data not shown).

Principal component analysis. Results of principal component analysis of eight body weights for 380 mice (185 males and 195 females) are shown in Table 3. The first two component axes were defined by scree tests. The first principal component (PC1) contained the majority of information from eight body weights, i.e., $80.5 \%$ of the total variance. All loadings for PC1 were positive in sign with relatively high values, extracting the so-called overall size factor. This indicated that PC1 approximated average growth, i.e., mice with extreme PC1 scores were either smaller or larger than the average at all ages examined. By the sign and magnitude of loadings, the second principal component (PC2) that accounted for $10.9 \%$ of the variance contrasted a middle growth phase from 3 to 6 weeks of age with a late growth phase from 6 to 10 weeks of age, an early growth phase from 1 to 3 weeks of age defined by Cheverud et al. (1996) was lacking in this study. Wt3 and Wt4 were predominant with the highest loadings in the middle growth phase, whereas Wt8 to Wt10 were dominant in the late growth phase. Very similar results were obtained in each sex (data not shown). These results showed that principal component analysis was able to extract a biologically meaningful essence from complex postnatal growth in body weight. Thus, we can treat $\mathrm{PC} 1$ and $\mathrm{PC} 2$ as univariate quantitative traits for QTL analyses below.

Interval mapping. As shown in Table 1, 41 of the 90 markers genotyped obviously had two or more alleles, whereas 32 markers fixed only one allele in the wild mice used here. For the remaining 17 markers, multiple alleles appeared to be seen, but their alleles were so close to one another that we could not separate them with absolute confidence on our electrophoresis condition. Accordingly, the 17 markers were treated as fixed markers.

For the 41 polymorphic markers, one-way ANOVA of PC1 and PC2 never evidenced significant associations between genotypes consisting of only wild mouse alleles at each marker locus and component scores in all sex groups at the experiment-wide $5 \%$ levels $(P<0.0012=$ $0.05 / 41$ ) based on the Bonferroni correction. Thus, we assumed that the wild mice were fixed for a single allele at all QTLs. Hence, two interval mappings, SIM and CIM, below were performed on a di-allelic system.

Results from the interval mapping analyses based on the sex-specific and sex-averaged linkage maps were not greatly different (data not shown). Using the sex-averaged map led to an easy comparison of QTL map positions between sexes to identify sex-specific QTLs (see below). Thus only results based on the sex-averaged map are reported here.

The empirical significance thresholds for SIM were determined by permutation and expressed as LOD scores. There were no obvious differences in estimated threshold values between two principal components and among three sex groups: $2.2-2.4$ at the genome-wide $10 \%$ levels, $2.5-2.7$ at the genome-wide $5 \%$ levels and $3.3-3.6$ at the genome-wide $1 \%$ levels.

Table 2. Means and standard deviations $(S D)$ in gram after correction for fixed and random effects and phenotypic correlations for body weights at 3-10 weeks after birth.

\begin{tabular}{cccccccccr}
\hline \hline Body weight $^{\mathrm{a}}$ & Mean $\pm S D$ & No. $^{\mathrm{b}}$ & Wt3 & Wt4 & Wt5 & Wt6 & Wt7 & Wt8 & Wt9 \\
\hline Wt3 & $12.8 \pm 0.9$ & 384 & & & & & & & \\
Wt4 & $14.3 \pm 1.2$ & 387 & 0.79 & & & & & & \\
Wt5 & $15.2 \pm 1.4$ & 385 & 0.68 & 0.84 & & & & & \\
Wt6 & $16.6 \pm 1.8$ & 387 & 0.59 & 0.72 & 0.88 & & & & \\
Wt7 & $16.2 \pm 1.8$ & 387 & 0.58 & 0.69 & 0.84 & 0.93 & & & \\
Wt8 & $16.6 \pm 2.0$ & 385 & 0.55 & 0.66 & 0.80 & 0.88 & 0.95 & & \\
Wt9 & $16.8 \pm 2.0$ & 387 & 0.55 & 0.64 & 0.78 & 0.86 & 0.93 & 0.96 & \\
Wt10 & $17.2 \pm 2.1$ & 387 & 0.55 & 0.61 & 0.73 & 0.82 & 0.90 & 0.94 & 0.96 \\
\hline
\end{tabular}

All correlations are highly significant at $P<0.0001$.

${ }^{a}$ Body weight at \# weeks of age.

${ }^{\mathrm{b}}$ No. of mice examined. 
Table 3. Loadings, eigenvalues and variances on the first two principal components (PC1 and PC2) for weight traits.

\begin{tabular}{ccc}
\hline \hline Body weight & PC1 & PC2 \\
\hline Wt3 & 0.718 & 0.588 \\
Wt4 & 0.820 & 0.485 \\
Wt5 & 0.913 & 0.187 \\
Wt6 & 0.938 & -0.072 \\
Wt7 & 0.959 & -0.186 \\
Wt8 & 0.948 & -0.250 \\
Wt9 & 0.941 & -0.269 \\
Wt10 & 0.916 & -0.278 \\
Eigenvalue & 6.44 & 0.87 \\
\% Variance & 80.5 & 10.9 \\
Cumulative \% & 80.5 & 91.4 \\
\hline
\end{tabular}

LOD score profiles for all chromosomes obtained by SIM analysis are depicted in Fig. 1. A total of seven QTLs affecting PC1 and PC2, significant at least at the genome-wide $10 \%$ levels, were mapped to chromosomes 2 , $5,6,10,13$, and X. The LOD score profiles for the four QTLs on chromosomes 5, 6 and X differed, obviously, between males and females. The peak LOD score exceeded the genome-wide $10 \%$ or less levels in one sex but did not in the other sex. The LOD score difference of $2.5-3.7$, corresponding to $P=0.00069-0.000037$, was significant at the Bonferroni-corrected 5\% level $(P<$ 0.005). These evidences suggested that the suggestive PC1 QTL on chromosome 5 was female-specific; and the two significant PC1 QTLs on X chromosome and the highly significant PC2 QTL on chromosome 6 were all male-specific. Except for the suggestive chromosome 5 QTL that mapped $1 \mathrm{cM}$ from the centromere with $5.9 \%$ of the total phenotypic variance, the other QTLs were all remapped by CIM or epistatic interaction analysis below.

Before CIM, the number of background markers to control for the genetic background was determined in each sex group separately. This number for PC1 and PC2 were, respectively, 4 and 1 in males, 6 and 2 in females and 9 and 4 in the sex-pooled group. The empirical significance thresholds estimated for CIM were not extremely different between two principal components and among three sex groups: $2.3-2.5$ at the genome-wide $10 \%$ levels, $2.6-2.8$ at the genome-wide $5 \%$ levels and 3.2 -3.7 at the genome-wide $1 \%$ levels.

The results of CIM analysis are shown in Table 4 and Fig. 2. A total of nine QTLs for PC1 and PC2 were detected on chromosomes 2, 4, 6, 7, 9, 10, 13 and X. As

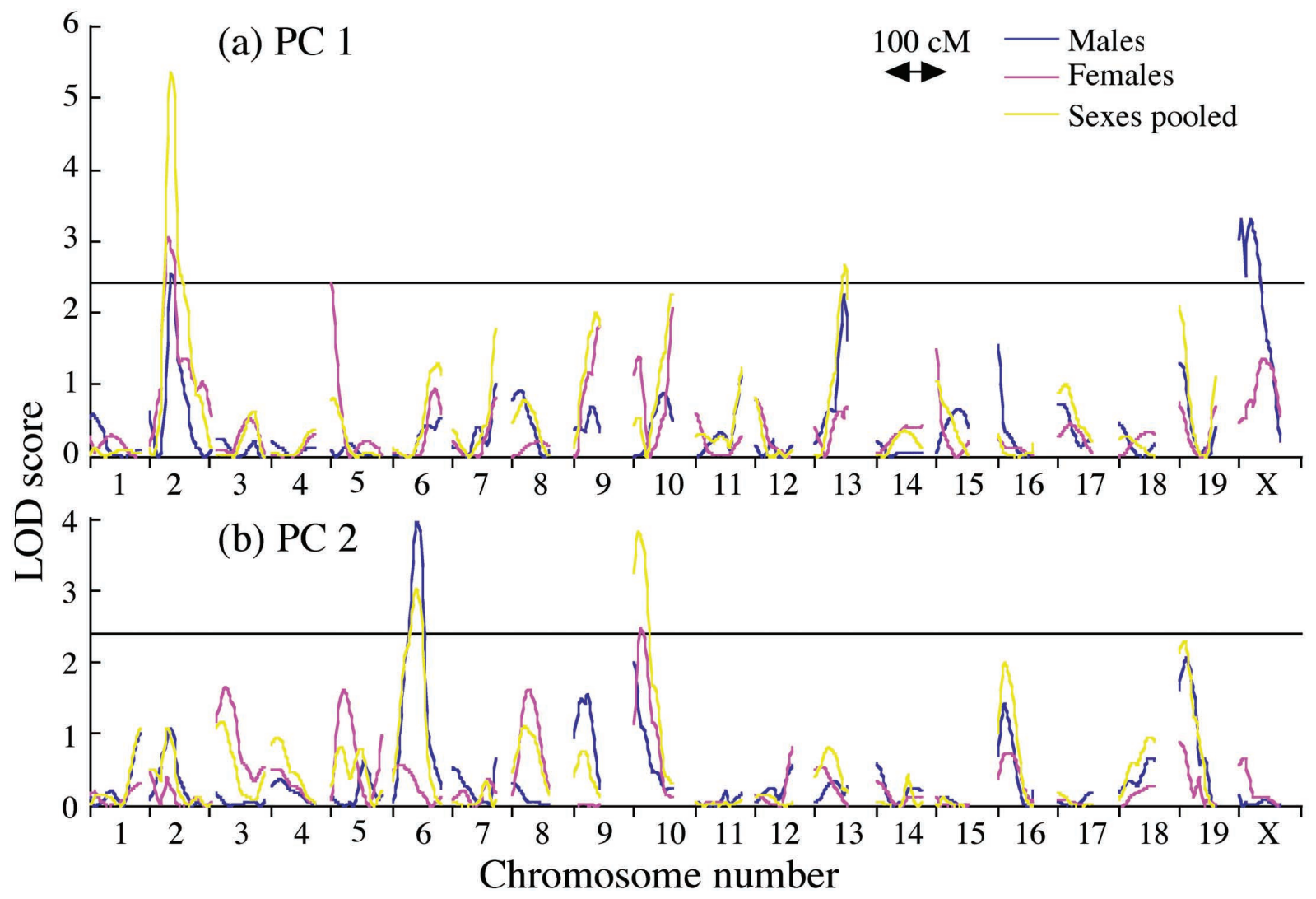

Fig. 1. LOD score profiles for all autosomes and $\mathrm{X}$ chromosome obtained from simple interval mapping (SIM) of the first two principal components (PC1 and PC2) by using QTL Cartographer software (Basten et al., 2000). The horizontal lines show the genome-wide $10 \%$ significance levels estimated by permutation tests. 
Table 4. Summary of QTLs detected by composite interval mapping (CIM) of the first two principal components (PC1 and PC2).

\begin{tabular}{|c|c|c|c|c|c|c|c|c|c|}
\hline \multirow[b]{2}{*}{ Trait } & \multirow{2}{*}{$\begin{array}{l}\text { QTL } \\
\text { designation }\end{array}$} & \multirow[b]{2}{*}{ Chr } & \multirow[b]{2}{*}{ Position $^{a}$} & \multirow[b]{2}{*}{$95 \% \mathrm{CI}^{\mathrm{b}}$} & \multirow[b]{2}{*}{ Effect $^{\mathrm{c}}$} & \multirow[b]{2}{*}{ Variance $^{\mathrm{d}}$} & \multicolumn{3}{|c|}{ LOD score ${ }^{\mathrm{e}}$} \\
\hline & & & & & & & Pooled & Male & Female \\
\hline \multirow[t]{7}{*}{$\mathrm{PC} 1$} & Pbwg1 & 2 & D2Mit324 + $2(35)$ & $26-44$ & 0.65 & 10.0 & $8.9 * * *$ & 2.2 & $4.1^{* * *}$ \\
\hline & Pbwg2 & 4 & D4Mit32 - $8(62)$ & $39-86$ & 0.41 & 3.9 & $2.9 * *$ & 0.4 & 1.5 \\
\hline & Pbwg3 & 7 & D7Mit259 + $0(72)$ & $40-104$ & 0.35 & 3.0 & $3.0 * *$ & 0.9 & 0.4 \\
\hline & $P b w g 4^{\mathrm{F}}$ & 9 & D9Mit18 + $0(71)$ & $46-96$ & 0.56 & 7.5 & 0.3 & 0.6 & $3.7^{* * *}$ \\
\hline & Pbwg5 & 10 & D10Mit145 - 2 (68) & $42-94$ & 0.39 & 3.7 & $3.4^{* *}$ & 0.2 & $2.5^{*}$ \\
\hline & Pbwg6 & 13 & D13Mit195 + $2(53)$ & $34-72$ & 0.46 & 5.2 & $4.9 * * *$ & $3.2^{* *}$ & 1.6 \\
\hline & $\operatorname{Pbwg} 7^{\mathrm{M}}$ & $\mathrm{X}$ & DXMit75 + 0 & $0-48$ & 0.53 & 6.9 & NA & $3.1^{* *}$ & 0.9 \\
\hline \multirow[t]{2}{*}{$\mathrm{PC} 2$} & $P b w g 8^{\mathrm{M}}$ & 6 & D6Mit29 - 5 (32) & $14-50$ & 0.68 & 12.1 & $2.7 * *$ & $4.0^{* * *}$ & 0.2 \\
\hline & Pbwg9 & 10 & D10Mit16 - 2 (14) & $0-36$ & 0.43 & 4.5 & $3.8^{* * *}$ & 0.5 & $2.8^{* *}$ \\
\hline
\end{tabular}

CIM was performed with QTL Cartographer software (Basten et al., 2000). The superscript letters M and F indicate male- and female- specific QTLs, respectively. Chr denotes chromosome.

${ }^{\text {a }}$ Position of QTL in cM from the nearest marker locus. The positive and negative signs indicate that the QTL maps \# cM, respectively, distal and proximal to the marker locus. The distance from the centromere is given in parentheses according to the mouse consensus map (MGD).

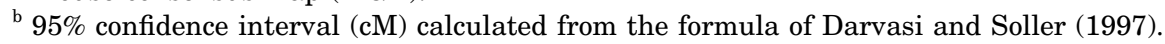

c Additive effect shown in phenotypic standard deviation unit.

${ }^{\mathrm{d}}$ Percentage of the phenotypic variance explained by the QTL.

${ }^{\text {e }}$ LOD scores were obtained by dividing likelihood ratio statistics by 4.605 in three sex groups (male, female and sexes pooled) separately. The parameter estimates of the locus were obtained from the sex group with the highest LOD score.

* Significant at the genome-wide $10 \%$ levels, ${ }^{* *}$ at the genome-wide $5 \%$ levels, $* * *$ at the genome-wide $1 \%$ levels, and NA, not applicable.

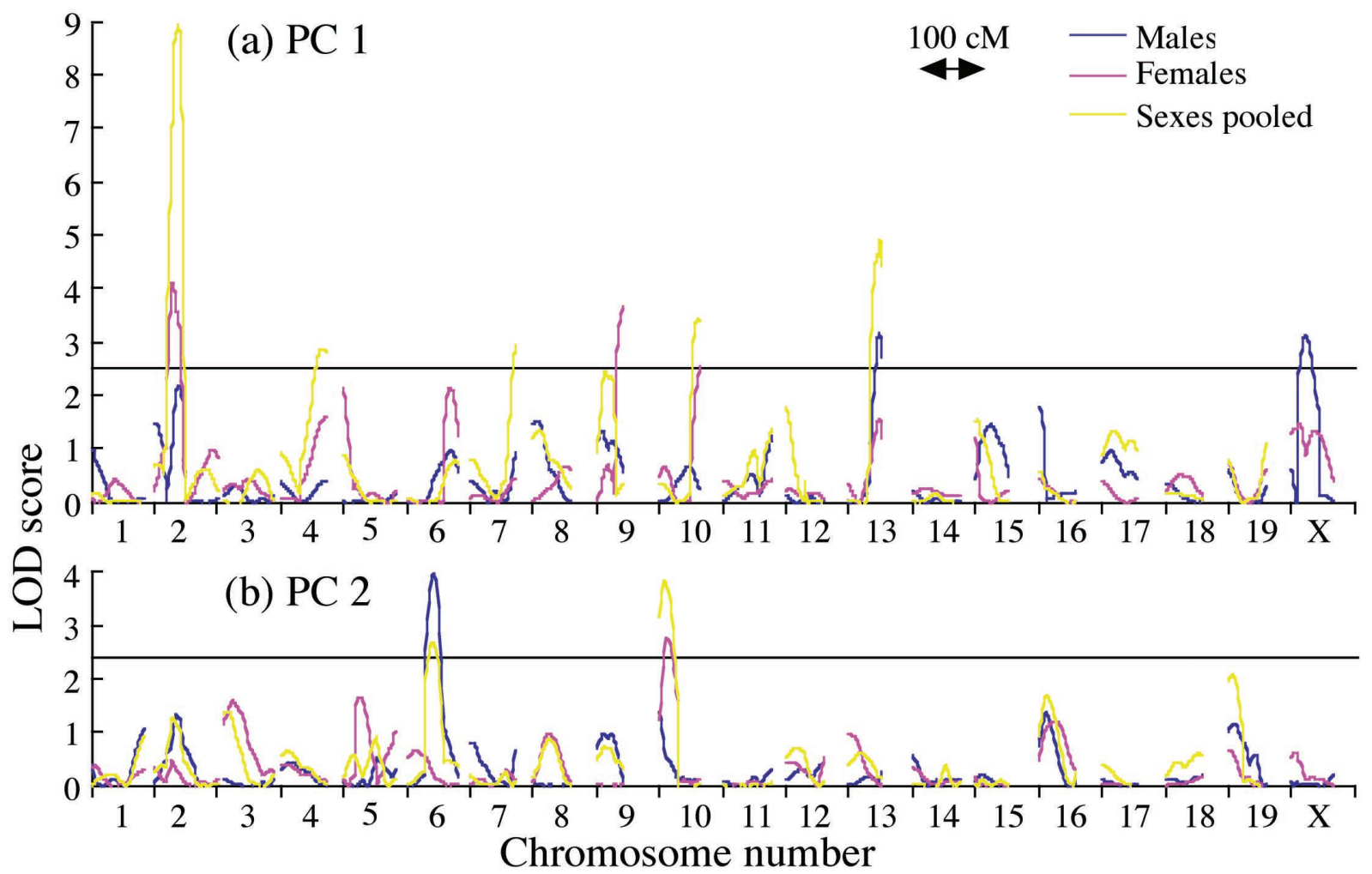

Fig. 2. LOD score profiles for all autosomes and $X$ chromosome obtained from composite interval mapping (CIM) of the first two principal components (PC1 and PC2) by using QTL Cartographer software (Basten et al., 2000). The horizontal lines show the genomewide $10 \%$ significance levels estimated by permutation tests. 
all QTLs exceeded the genome-wide 5\% or less levels, we assigned a series of gene symbols from Pbwg1 (Postnatal body weight growth 1) to Pbwg9 to the QTLs. Of these, four PC1 QTLs, Pbwg2-Pbwg5, were newly uncovered by CIM. The remaining PC1 and PC2 QTLs were identified by the above SIM analysis. For Pbwg4, the LOD score difference between two sexes was 3.2. Moreover, the sex difference in LOD score profiles made it clear that this QTL had a female-specific effect on PC1. Since PC1 summarized information on the entire growth process as described earlier (Table 3), all the PC1 alleles derived from wild mice reduced growth. By contrast, the analyses of PC2 clarified that Pbwg8 and Pbwg9 exerted their effects in the middle or earlier growth phase but the alleles did not describe the direction of growth like PC1 because PC2 specified only the growth phase where the QTLs had the largest impact (Table 3). Individual QTLs explained $3.9-10.9 \%$ of the phenotypic variance (Table 4). The total contribution of all identified main-effect QTLs to the phenotypic variance were $36.4 \%$ in males and $40.8 \%$ in females for $\mathrm{PC} 1$ and $16.2 \%$ and $9.2 \%$ in the respective sexes for $\mathrm{PC} 2$.

Interestingly, comparison of LOD score profiles between SIM and CIM (Figs. 1 and 2) revealed that QTLs greatly contributing to $\mathrm{PC} 1$ appeared to be $P b w g 6$ and male-specific Pbwg7 in males and Pbwg1 and female-specific Pbwg4 in females. Likewise, loci mostly responsible for PC2 seemed to be male-specific Pbwg8 in males and
Pbwg9 in females. In addition, combined results from SIM and CIM provided a possibility that Pbwg6 and Pbwg9 may be male- and female-specific, respectively.

Epistatic interaction. As described earlier, two-stage tests were used to declare significant epistatic interactions. The permutation test of Map Manager QTX provided genome-wide $63 \%, 5 \%$ and $0.1 \%$ significance thresholds for testing the significance of overall effects obtained from pairwise comparisons among all markers (Chmielewicz and Manly 2002), but we adopted only the genome-wide 5\% thresholds because of consistency with those levels used for the above SIM and CIM. The 5\% threshold levels, expressed as LOD scores and $P$ values in parentheses, was determined to be $5.2-$ $5.4\left(1.8-2.4 \times 10^{-5}\right)$ for PC1 and $5.2\left(2.5-2.9 \times 10^{-5}\right)$ for PC2 in three sex groups. To test the significance of the marker by marker interaction, the genome-wide thresholds computed for SIM were used as approximates and their converted $P$ values were $7.6 \times 10^{-4}-1.4 \times 10^{-3}, 3.7$ $-7.0 \times 10^{-4}$ and $5.6 \times 10^{-5}-1.2 \times 10^{-4}$ at the genome-wide 10,5 and $1 \%$ levels, respectively. The Bonferroni-corrected experiment-wise $5 \%$ level $(P<0.05 / 18=0.0028)$ were also employed.

Pairwise testing across all 90 markers with Map Manager QTX revealed a total of 18 marker pairs with significant overall effects on either principal components in all three sex groups. Of these, only five marker pairs

Table 5. $\quad P$ values of significant epistatic interactions between marker pairs.

\begin{tabular}{|c|c|c|c|c|c|c|c|}
\hline Trait & Chrs & Marker 1 & Marker 2 & Sex & Overall & Marker $1 \times$ marker 2 & Marker $1 \times$ marker $2 \times$ sex $^{a}$ \\
\hline \multirow[t]{9}{*}{$\mathrm{PC} 1$} & 2 and 9 & D2Mit48 & D9Mit18 & Pooled & $1.8 \times 10^{-5 * *}$ & $3.3 \times 10^{-4 * *}$ & 0.99 \\
\hline & & $($ Pbwg11) & $(P b w g 4)$ & Male & $0.05<$ & NA & \\
\hline & & & & Female & $1.8 \times 10^{-4}$ & 0.0086 & \\
\hline & 2 and 12 & D2Mit324 & D12Mit4 & Pooled & $2.3 \times 10^{-7}$ & 0.0047 & 0.0064 (male specific) \\
\hline & & $($ Pbwg1) & $(P b w g 12)$ & Male & $9.1 \times 10^{-6 * * *}$ & $1.6 \times 10^{-4 * *}$ & \\
\hline & & & & Female & $0.05<$ & NA & \\
\hline & 6 and $\mathrm{X}$ & D6Mit74 & DXMit124 & Pooled & $1.2 \times 10^{-4}$ & 0.0073 & 0.0064 (male specific) \\
\hline & & (Pbwg8) & $($ Pbwg10) & Male & $2.6 \times 10^{-6 * *}$ & $1.3 \times 10^{-4 * *}$ & \\
\hline & & & & Female & $0.05<$ & NA & \\
\hline \multirow[t]{6}{*}{$\mathrm{PC} 2$} & 5 and 10 & D5Mit51 & D10Mit16 & Pooled & $0.05<$ & NA & 0.015 \\
\hline & & $($ Pbwg13) & $(P b w g 9)$ & Male & $0.05<$ & NA & \\
\hline & & & & Female & $2.5 \times 10^{-6 * *}$ & $4.5 \times 10^{-4 * *}$ & \\
\hline & 6 and 13 & D6Mit29 & D13Mit26 & Pooled & $4.9 \times 10^{-6 * *}$ & $8.2 \times 10^{-4 *}$ & 0.61 \\
\hline & & (Pbwg8) & (Pbwg6) & Male & $9.5 \times 10^{-5 * *}$ & 0.0052 & \\
\hline & & & & Female & $0.05<$ & NA & \\
\hline
\end{tabular}

All possible pairwise comparisons between 90 markers (Table 1) were performed with Map Manager QTX software (Manly et al. 2001) on the assumption that an epistatic QTL is at a marker locus. The obtained likelihood ratio statistics for the overall and marker 1 by marker 2 interaction effects were converted into $P$ values by using a $\chi^{2}$ distribution with one or three degrees of freedom. QTL designation was shown in parentheses.

a Tested by three-way ANOVA. If the marker 1 by marker 2 by sex interaction was significant at the Bonferroni-corrected experiment-wise $5 \%$ level $(P<0.05 / 5=0.01)$, the direction of sex specificity was shown in parentheses.

* Significant at the genome-wide $10 \%$ levels, ${ }^{* *}$ at the genome-wide $5 \%$ levels, and NA, not applicable. 

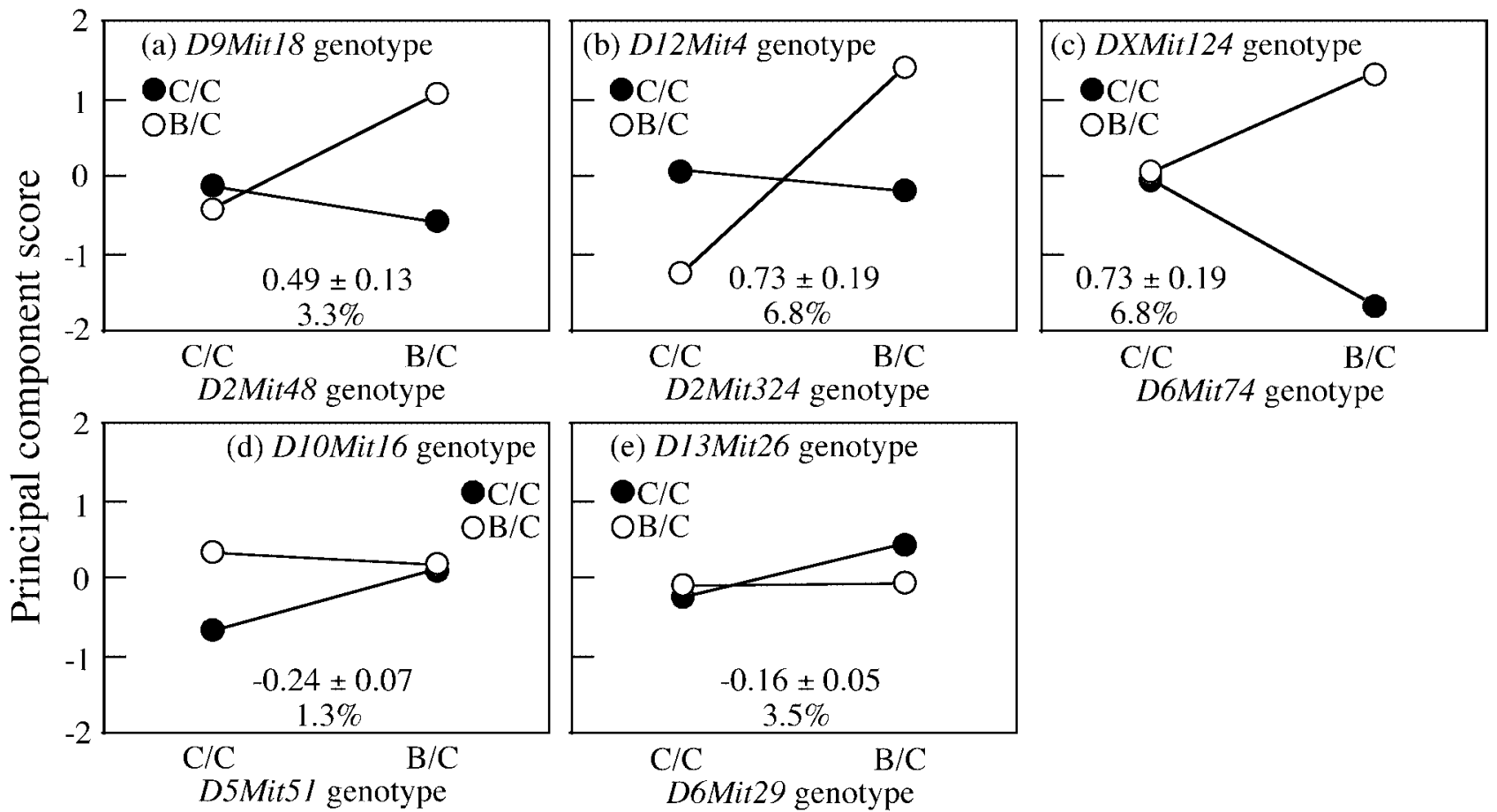

D6Mit74 genotype

Fig. 3. Direction of effects of epistatic interactions between marker pairs. The number shows the mean and standard error (SE) of the marker by maker interaction effect estimated by two-way ANOVA, together with the percentage of the phenotypic variance explained by the interaction. B and C denote the marker alleles derived from C57BL/6J and wild mice, respectively. The $S E$ of each point ranged from 0.1 to 0.4 .

showed significant marker by marker interactions at the genome-wide $10 \%$ or less levels as well as the Bonferronicorrected $5 \%$ level. The $P$ values for these five pairs are indicated in Table 5. Three-way ANOVA revealed that two of the five marker pairs obviously had significant male-specific effects on PC1 at the Bonferroni-corrected $5 \%$ level $(P<0.05 / 5=0.01)$. The interaction of the marker pair D5Mit51 and D10Mit16 with sex did not somewhat pass the significant threshold. But this pair was very likely to be female-specific as neither male nor pooled data showed significant overall effects and marker by marker effects.

The direction of the phenotypic effects of the five marker pairs is depicted in Fig. 3. The effect of the marker by marker interaction estimated by two-way ANOVA ranged from -0.24 to 0.73 . Surprisingly the sign of the effects was positive for the three marker pairs affecting PC1 (Figs. 3a to 3c), although negative effects were generally expected because of the wild mice with about $60 \%$ lower adult body weight than C57BL/6J (Ishikawa et al. 2000). When one marker locus was homozygous for the wild mouse allele (C), C/C homozygotes at another marker locus had a tendency to be higher in principal component score than B/C heterozygotes. This indicated that the epistatic interaction effects unexpectedly enhanced growth. By contrast, negative effects were seen for the remaining two marker pairs affecting PC2 (Figs. 3d and 3e). Individual marker pairs explained $1.3-6.8 \%$ of the phenotypic variance. Taken together, epistasis accounted for $16.7 \%$ in males and $2.9 \%$ in females for PC1 and $3.5 \%$ and $5.5 \%$ for PC2, respectively.

Comparison of results from the epistatic interaction analysis with those from the SIM and CIM analyses indicated that there were at least nine epistatic QTLs. The QTLs marked by two markers D6Mit74 and D6Mit29 on chromosome 6 were likely to be the same locus, i.e., Pbwg8, judging from the map position and 95\% confidence interval (Table 4). Likewise, the map positions of four QTLs marked by D2Mit324, D9Mit18, D10Mit16 and D13Mit26 were nearly identical with those of main effect QTLs discovered by CIM (Table 4), meaning that they had both main effects and epistatic interaction effects. The QTL marked by DXMit124 was not confirmed by CIM (Table 4) but was done by SIM (Fig. 1). However, the remaining three QTLs marked by D2Mit48, D12Mit4 and D5Mit51 mapped to chromosomal regions where no maineffect QTLs were identified (Table 4, Figs. 1 and 2), indicating that these three QTLs had only epistatic interaction effects. We thus gave the three new QTLs continuous gene symbols as shown in Table 5 .

\section{DISCUSSION}

By using principal component analysis in a genomewide QTL scan, we were able to map eight significant 
QTLs with main effects on PC1 (Pbwg1-Pbwg7 and Pbwg10) to chromosomes 2, 4, 7, 9, 10, 13 and $\mathrm{X}$ and two significant main-effect QTLs for PC2 (Pbwg8 and Pbwg9) to chromosomes 6 and 10. The eight PC1 QTLs were involved in pathways regulating the entire postnatal growth process, whereas the two PC2 QTLs functioned mainly in the middle or earlier growth phase. Furthermore, we revealed that the detected six main-effect QTLs (Pbwg1, Pbwg4, Pbwg6, and Pbwg8-Pbwg10) interacted epistically with one another or the other three new QTLs without main effects (Pbwg11-Pbwg13) on chromosomes 2,5 and 12 . Our previous mapping study on adult body weight at 10 weeks of age in the same population identified six significant main-effect QTLs (C10bw1 to C10bw6) on chromosomes 2, 9, 11, 13 and $\mathrm{X}$ and one significant epistatic QTL $(C 10 b w 7)$ on chromosome 12 (Ishikawa et al. 2000). Of these, C10bw1 on chromosome 2, C10bw5 on chromosome 13 and C10bw 7 on chromosome 12 are identical with, respectively, Pbwg1, Pbwg6 and Pbwg12. We thus change the previous gene symbols of the three loci into present ones for consistency.

Additional QTLs may have been overlooked in the present genome-wide QTL scan using the wild mice that are genetically heterogeneous, with some reasons. We performed QTL analyses on the assumption that QTL alleles fixed in the wild mice because there was no statistical evidence for any associations of marker alleles segregating in the wild mice and principal components. However, it is thought to be very natural that the wild mice have multiple alleles at some QTLs. When such multiple alleles from the wild mice have different phenotypic effects, the allele-averaged effect is subjected to QTL mapping with a di-allelic system. This can obviously lead to reduce the contrast between alleles from the C57BL/6J and wild mice and hence the power of QTL detection. Thus, even if QTLs were detected, the effects of the wild mouse alleles estimated here may not always reflect the true ones. The precise estimation of each allele effect can be achieved by construction and investigation of congenic strains. The other general causes to overlook QTLs, such as type of a mapping population and marker density, are described in our previous study (Ishikawa et al. 2000).

Some definitive advantages and disadvantages of using principal component analysis in a QTL mapping study in which many traits are recorded are described in detail by Andersson-Eklund et al. (2000) and Mähler et al. (2002). Briefly, if QTLs affecting different principal components are located on the same chromosomal region, they must be linked loci because of statistical uncorrelation between the components. An example of this is our Pbwg5 and Pbwg9 on chromosome 10, but they were not so tightly linked. Furthermore, the analyses of principal components may better approximate the assumption of normally distributed phenotypes required for most tradi- tional QTL mapping methods, which some separate analyses of original measurements may violate it. A possible disadvantage is that the magnitudes of the estimated effects are difficult to interpret directly in terms of traits. In the present study, this is the case with QTLs affecting PC2, although the result from the PC1 analysis is straightforwardly understandable. If the original estimates are needed, then they can be obtained either by individual single-trait analyses at the positions of the QTLs mapped or by a back transformation of the estimated effects.

Recently, epistasis has been evaluated experimentally and revealed to be an important contributor to quantitative traits (Mackay 2001). In mice, $F_{2}$ populations from a cross of LG/J and SM/J (Routman and Cheverud 1997, Cheverud et al. 2001) and from a cross of DU6i and DBA/ 2 (Brockmann et al. 2000) have been used for genomewide search for epistatic QTLs affecting postnatal growth and obesity traits. These studies revealed that almost every QTLs identified participate in some epistatic interactions with relatively small individual effects. Such a tendency is observed in our study. Six of the 10 maineffect QTLs identified here participated in epistatic interactions. Interestingly, Pbwg6 with a main effect on PC1 had an epistatic interaction effect on a different trait, PC2. Uniquely, Pbwg8 with a main effect on PC2 exerted its interaction effect on both principal components. As the interaction effect of one QTL on different traits are reported (Brockmann et al. 2000, Cheverud et al. 2001), these two QTLs may potentially have pleiotropic effects on different principal components that are probably regulated by distinct pathways of postnatal growth because of uncorrelation between the two components. But we could not rule out a possibility that each of the QTLs was consisted of tightly linked loci, because two linked QTLs, approximately $0.3 \mathrm{cM}$ apart, are reported to operate in different developmental pathways of sugar content in tomato (Fridman et al. 2002).

Our epistatic QTL Pbwg11 on chromosome 2 maps 15 $\mathrm{cM}$ distal to Bgeq2 for body weight at 10 weeks of age (Routman and Cheverud 1997), although the former doe not have a main effect and the later does it. Bw13 on chromosome 5 with a main effect on body weight at 6 weeks of age (Brockmann et al. 2000) maps very close to our Pbwg 13 without a main effect, while another QTL on chromosome 9 without a main effect (Brockmann et al. 2000) maps $10 \mathrm{cM}$ proximal to Pbwg4 with a main effect. In addition to the difference in direct effects on the phenotypes, counterpart loci interacting with these QTLs are located on quite different chromosomes. Furthermore, Brockmann et al. (2000) reported that the total contribution of epistasis to the phenotypic variance of 6week body weight is estimated to be $33 \%$ and that of all identified QTLs with main effects and epistatic interaction effects accounts for $65 \%$ of the variance by multiple 
regression analyses using the nearest markers of QTL positions. Our corresponding values were estimated to be roughly $10 \%$ and $35 \%$ for $\mathrm{PC} 1$ and $5 \%$ and $13 \%$ for PC2. The lower contributions of our QTLs may largely be caused by use of the genetically heterogeneous wild mice for construction of the mapping population and of principal component analysis for detection of major QTLs underlying postnatal growth, both leading to reduction of the power to detect QTLs as described earlier.

We found that about half the mapped QTLs with main and/or epistatic effects on body weight growth are sexspecific. For main effect QTLs, several recent studies reported their sex-specific effects on body weight and adiposity in mice. Three male-specific QTLs on X chromosome, one is $B w 12$ for body weight at 6 weeks of age (Brockmann et al. 1998) and the others are $B w 1$ and $B w 2$ for 40-week body weight (Dragani et al. 1995), are detected in the regions containing our male-specific QTLs, Pbwg7 and Pbwg10. Obq6 with a male-specific effect on adiposity index (Taylor et al. 1999) is also reported in those regions. Male specific $S k l 3$ affecting both tail length and 10-week body weight (Cheverud et al. 2001) maps close to our male-specific Pbwg9 on chromosome 6 . On the other hand, fewer studies have so far investigated sex-specific effects of epistatic interactions for body weight and its related traits in mice. Only Cheverud et al. (2001) reported that about half the QTL combinations detected have male- or female-specific effects on adiposity and tail length. Enough evidence for sex-specific epistatic interactions has not been accumulated like this, whereas sex-specific QTLs with main effects have commonly been reported on different quantitative traits in mice (e.g., Farmer et al. 2001, Mähler et al. 2002, Butterfield et al. 2003) and other animals (Mackay 2001). Thus, sex specificity must be a general property of genes underlying variation in quantitative traits.

The mechanisms underlying sex-specific QTLs have remained unclear. But our sex-specific QTLs may be dependent on the presence of sex hormones or may be influenced by genes on $\mathrm{Y}$ chromosome. Castration experiments in OLETF rats exhibiting an extreme sex difference in incidence of diabetes mellitus (more than $90 \%$ in males and $0 \%$ in females by 6 months of age) indicate that the development of diabetes is reduced in males but enhanced in females, and the same tendency is observed for body weight changes in the castrated rats (Shi et al. 1994). Not related to growth phenotypes, but there is a good example of the influence of Y-linked genes. The Yaa gene is known to interact with susceptibility loci on autosomes to accelerate the development of spontaneous lupus and lymphoproliferation in male mice (Butterfield et al. 2003). Further studies using congenic strains will be needed to determine the physiological bases of our sexspecific QTL effects.
A great number of QTLs affecting growth and growthrelated traits have so far been reported in the literature (e.g., Dragani et al. 1995, Cheverud et al. 1996, Keightley et al. 1996, Brockmann et al. 1998, Morris et al. 1999, Taylor et al. 1999, Vaughn et al. 1999, Brockmann et al. 2000, Cheverud et al. 2001, Danielle et al. 2003). When we made comparisons of QTL map positions between the present and other studies, a number of the previously reported QTLs could be found within the 95\% confidence intervals of our QTLs as described in some comparisons above, and some QTLs are likely to be the same loci. As the confidence intervals obtained from genome-wide QTL analysis of this level are surely very broad, such comparisons will be problematic and questionable. Keightley and Knott (1999) have shown that getting a statistically significant concordance between the positions of QTLs in two different mapping experiments, i.e., the case that the same QTLs are segregating in the two crosses, would be unlikely if the trait variation is explained by a large number of QTLs and the experimental populations are not closely related. As M. m. castaneus wild mice caught in the Philippines were employed to construct our QTL mapping population, many QTLs detected here appear to be novel or, even though the positions of QTLs are same between the two crosses, most of our QTL alleles may be new.

As a lot of growth-related functional genes are listed in MGD, it could be possible to find potential candidate genes for our QTLs. We here describe the most obvious candidates for our QTLs. Pbwg1 on chromosome 2 locates in the vicinity of $\mathrm{Gcg}$. Candidates for Pbwg3 on chromosome 7 are Tub, Ucp2 and Ucp3. Ghrhr and Igf1 are located in the confidence intervals of Pbwg8 on chromosome 6 and Pbwg5 on chromosome 10, respectively. Fine-scale mapping will be required to reduce the number of candidate genes and to identify any of the candidate genes actually contributing to our QTL effects.

We gratefully acknowledge Dr. Yoshitaka Nagamine (National Institute of Livestock and Grassland Science) for a critical discussion on the manuscript and Sayuri Hatada for assistance with genotyping.

\section{REFERENCES}

Andersson-Eklund, L., Uhlhorn, H., Lundeheim, N., and Dalin, G. (2000) Mapping quantitative trait loci for principal components of bone measurements and osteochondrosis scores in a wild bore $\times$ Large White intercross. Genet. Res. 75, 223-230.

Andersson, L. (2001) Genetic dissection of phenotypic diversity in farm animals. Nat. Rev. Genet. 2, 130-138.

Basten, C. J., Weir, B. S., and Zeng, Z.-B. (2000) QTL Cartographer, Version 1.14. Department of Statistics, North Carolina State University, Raleigh, NC.

Bergeson, S. E., Warren, R. K., and Crabbe, J. C., Metten, P., Erwin, V. G., and Belknap, J. K. (2003) Chromosomal loci influencing chronic alcohol withdrawal severity. Mamm. 
Genome. 14, 454-463.

Brockmann, G. A., Haley, C. S., Renne, U., Knott, S. A., and Schwerin, M. (1998) Quantitative trait loci affecting body weight and fatness from a mouse line selected for extreme high growth. Genetics 150, 369-381.

Brockmann, G. A., Kratzsch, J., Haley, C. S., Renne, U., Schwerin, M., and Karle, S. (2000) Single QTL effects, epistasis, and pleiotropy account for two-thirds of the phenotypic F2 variance of growth and obesity in DU6i $\times$ DBA/2 mice. Genome Res. 10, 1941-1957.

Butterfield, R. J., Roper, R. J., Rhein, D. M., Melvold, R. W., Haynes, L., Ma, R. Z., Doerge, R. W., and Teuscher, C. (2003) Sex-specific quantitative trait loci govern susceptibility to Theiler's murine encephalomyelitis virus-induced demyelination. Genetics 163, 1041-1046.

Chase, K., Carrier, D. R., Adler, F. R., Jarvik, T., Ostrander, E. A., Lorentzen, T. D., and Lark, K. G. (2002) Genetic basis for systems of skeletal quantitative traits: principal component analysis of the canid skeleton. Proc. Natl. Acad. Sci. USA 99, 9930-9935.

Cheverud, J. M., Routman, E. J., Duarte, F. A. M., van Swinderen, B., and Cothran, K. (1996) Quantitative trait loci for murine growth. Genetics 142, 1305-1319.

Cheverud, J. M. , Vaughn, T. T., Pletscher, L. S., Peripato, A. C., Adams, E. S., Erikson, K. J., and King-Ellison, K. J. (2001) Genetic architecture of adiposity in the cross of LG/J and SM/J inbred mice. Mamm. Genome 12, 3-12.

Chmielewicz, K. M., and Manly, K. F. (2002) User Manual for QTX, revised for QTXb17. Roswell Park Cancer Institute, Buffalo, NY.

Churchill, G. A., and Doerge, R. W. (1994) Empirical threshold values for quantitative trait mapping. Genetics 138, 963971.

Darvasi, A., and Soller, M. (1997) A simple method to calculate resolving power and confidence interval of QTL map location. Behav. Genet. 27, 125-132.

Dillon, W. R., and Goldstein, M. (1984) Multivariate Analysis: Methods and Applications. John Wiley \& Sons, New York.

Doerge, R. W. (2002) Mapping and analysis of quantitative trait loci in experimental populations. Nat. Rev. Genet. 3, 4352.

Doerge, R. W., Zeng, Z.-B., and Weir, B. S. (1997) Statistical issues in the search for genes affecting quantitative traits in experimental populations. Stat. Sci. 12, 195-219.

Dragani, T. A., Zeng, Z.-B., Canzian, F., Gariboldi, M., Ghilarducci, M. T., Manenti, G., and Pierotti, M. A. (1995) Mapping of body weight loci on mouse chromosome X. Mamm. Genome 6, 778-781.

Farmer, M. A., Sundberg J. P., Bristol, I. J., Churchill, G. A., Li, R., Elson, C. O., and Leiter, E. H. (2001) A major quantitative trait locus on chromosome 3 controls colitis severity in IL-10-deficient mice. Proc. Natl. Acad. Sci. USA 98, 13820-13825.

Flint, J., and Mott, R. (2001) Finding the molecular basis of quantitative traits: successes and pitfalls. Nat. Rev. Genet. 2, 437-445.

Frary, A., Nesbitt, T. C., Esbitt, A., Frary, A., Grandillo, S., van der Knaap, E., Cong, B., Lui, J., Meller, J., Elber, R., Aipert, K. B., and Tanksley, S. D. (2000) fw2.2: a quantitative trait locus key to the evolution of tomato fruit size. Science $\mathbf{2 8 9}$, $85-88$.

Fridman, E., Liu, Y. S., Carmel-Goren, L., Gur, A., Shoresh, M., Pleban, T., Eshed, Y., and Zamir, D. (2002) Two tightly linked QTLs modify tomato sugar content via different physiological pathways. Mol. Genet. Genomics 266, 821-
826.

Grisart, B., Coppieters, W., Farnir, F., Karim, L., Ford, C., Berzi, P., Cambisano, N., Mni, M., Reid, S., Simon, P. Spelman, R., Georges, M., and Snell, R. (2001) Positional candidate cloning of a QTL in dairy cattle: identification of a missense mutation in the bovine DGAT1 gene with major effect on milk yield and composition. Genome Res. 12, $222-231$.

Guenet, J., and Bonhomme, F. (2003) Wild mice: an everincreasing contribution to a popular mammalian model. Trends Genet. 19, 24-31.

Ishikawa, A., Matsuda, Y., and Namikawa, T. (2000) Detection of quantitative trait loci for body weight at 10 weeks from Philippine wild mice. Mamm. Genome 11, 824-830.

Keightley, P. D., Hardge, T., May, L., and Bulfield, G. (1996) A genetic map of quantitative trait loci for body weight in the mouse. Genetics 142, 227-235.

Keightley, P. D., and Knott, S. A. (1999) Testing the correspondence between map positions of quantitative trait loci. Genet. Res. 74, 323-328.

Knott, S. A., Marklund, L., Haley, C. S., Andersson, K., Davies, W., Ellegren, H., Fredholm, M., Hansson, I., Hoyheim, B. Lundstrom, K., Moller, M., and Andersson, L. (1998) Multiple marker mapping of quantitative trait loci in a cross between outbred wild boar and Large White pigs. Genetics 149, 1069-1080.

Lander, E. S., and Botstein, D. (1989) Mapping Mendelian factors underlying quantitative traits using RFLP linkage maps. Genetics 121, 184-199.

Liang, T., Spence, J., Liu, L., Strother, W. N., Chang, H. W., Ellison, J. A., Lumeng, L., Li, T.-K., Foroud, T., and Carr, L. G. (2003) $\alpha$-synuclein maps to a quantitative trait locus for alcohol preference and is differentially expressed in alcoholpreferring and -nonpreferring rats. Proc. Natl. Acad. Sci. USA 100, 4690-4695.

Mackay, T. F. C. (2001) Quantitative trait loci in Drosophila. Nat. Rev. Genet. 2, 11-20.

Mähler, M., Most, C., Schmidtke, S., Sundberg, J. P., Li, R., Hedrich, H. J., and Churchill, G. A. (2002) Genetics of colitis susceptibility in IL-10-deficient mice: backcross versus F2 results contrasted by principal component analysis. Genomics 80, 274-282.

Mangin, B., Thoquet, P., and Grimsley, N. (1998) Pleiotropic QTL analysis. Biometrics 54, 88-99.

Manly, K. F., Cudmore Jr, R. H., and Meer, J. M. (2001) Map Manager QTX, cross-platform software for genetic mapping. Mamm. Genome 12, 930-932.

Mather, K., and Jinks J. L. (1977) Introduction to Biometrical Genetics. Chapman and Hall, London.

Morris, K. H., Ishikawa, A., and Keightley, P. D. (1999) Quantitative trait loci for growth traits in C57BL/6J X DBA/2J mice. Mamm. Genome 10, 225-228.

Mouse Genome Database (MGD) (2003) Mouse Genome Informatics Web Site, The Jackson Laboratory, Bar Harbor, Maine. World Wide Web (URL: http://www.informatics.jax.org/). (July 2003).

Nishioka, Y. (1995) The origin of common laboratory mice. Genome 38, 1-7.

Quintanilla, R., Milan, D., and Bidanel, J.-P. (2002) A further look at quantitative trait loci affecting growth and fatness in a cross between Meishan and Large White pig populations. Genet. Sel. Evol. 34, 193-210.

Rance, K. A., Hill, W. G., Keightley, P. D. (1997a) Mapping quantitative trait loci for body weight in the $\mathrm{X}$ chromosome in mice. I. Analysis of a reciprocal $\mathrm{F}_{2}$ population. Genet. 
Res. 70, 117-124. Res. 70, 117-124.

Rance, K. A., Heath, S. C., and Keightley, P. D. (1997b) Mapping quantitative trait loci for body weight in the $\mathrm{X}$ chromosome in mice. II. Analysis of congenic backcrosses. Genet. Res. 70, 125-133.

Reed, D. R., Li, X., McDaniel, A. H., Lu, K., Li, S., Tordoff, M. G., Price, R. A., and Bachmanov, A. A. (2003) Loci on chromosomes 2, 4, 9, and 16 for body weight, body length, and adiposity identified in a genome scan of an $\mathrm{F}_{2}$ intercross between the 129P3/J and C57BL/6ByJ mouse strains. Mamm. Genome 14, 302-313.

Routman, E., and Cheverud, J. (1994) A rapid method of scoring simple sequence repeat polymorphisms with agarose gel electrophoresis. Mamm. Genome 5, 187-188.

Routman, E. J., and Cheverud, J. M. (1997) Gene effects on a quantitative trait: two-locus epistatic effects measured at microsatellite markers and at estimated QTL. Evolution 51, 1654-1662.

Sen, S, and Churchill, G. A. (2001) A statistical framework for quantitative trait mapping. Genetics 159, 371-387.

Shi, K., Mizuno, A., Sano, T., Ishida, K., and Shima, K. (1994) Sexual difference in the incidence of diabetes mellitus in Otsuka-Long-Evans-Tokushima-Fatty rats: effects of castra- tion and sex hormone replacement on its incidence. Metabolism 43, 1214-1220.

Taylor, B. A., Tarantino, L. M., and Phillips, S. J. (1999) Genderinfluenced obesity QTLs identified in a cross involving the KK type II diabetes-prone mouse strain. Mamm. Genome 10, 963-968.

Vaughn, T. T., Pletscher, L. S., Peripato, A., King-Ellison, K., Adams, E., Erikson, C., and Cheverud, J. M. (1999) Mapping quantitative trait loci for murine growth: a closer look at genetic architecture. Genet. Res. 74, 313-322.

Weller, J. I., Wiggans, G. R., van Raden, P. M., and Ron, M. (1996) Application of a canonical transformation to detection of quantitative trait loci with the aid of genetic markers in multitrait experiments. Theor. Appl. Genet. 92, 9981002.

Zeng, Z.-B. (1993) Theoretical basis for separation of multiple linked gene effects in mapping quantitative trait loci. Proc. Natl. Acad. Sci. USA 90, 10972-10976.

Zeng, Z.-B. (1994) Precision mapping of quantitative trait loci. Genetics 136, 1457-1468.

Zeng, Z.-B., Kao, C., and Basten, C. J. (1999) Estimating the genetic architecture of quantitative traits. Genet. Res. 74, 279-289. 